\title{
Heat-Killed Lactobacillus plantarum KCTC 13314BP Enhances Phagocytic Activity and Immunomodulatory Effects via Activation of MAPK and STAT3 Pathways
}

\author{
Minju Jeong ${ }^{1 \dagger}$, Jae Hwan Kim ${ }^{1 \dagger}$, Hee Yang ${ }^{1,2}$, Shin Dal Kang ${ }^{3}$, Seongbong Song ${ }^{3}$, Deukbuhm Lee ${ }^{3}$, Ji Su Lee ${ }^{4}$, \\ Jung Han Yoon Park ${ }^{5}$, Sanguine Byun ${ }^{4 *}$, and Ki Won Lee ${ }^{1,2,5 *}$ \\ ${ }^{1}$ Department of Agricultural Biotechnology, Seoul National University, Seoul 08826, Republic of Korea \\ ${ }^{2}$ Advanced Institutes of Convergence Technology, Seoul National University, Suwon 16229, Republic of Korea \\ ${ }^{3}$ Research Institute of Food and Biotechnology, SPC Group, Seoul 151742, Republic of Korea \\ ${ }^{4}$ Division of Bioengineering, Incheon National University, Incheon 22012, Republic of Korea \\ ${ }^{5}$ Research Institute of Agriculture and Life Sciences, Seoul National University, Seoul 08826, Republic of Korea
}

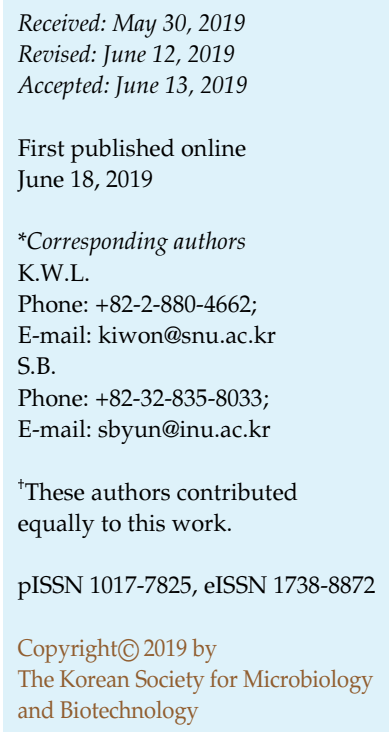

Identification of novel probiotic strains is of great interest in the field of functional foods. Specific strains of heat-killed bacteria have been reported to exert immunomodulatory effects. Herein, we investigated the immune-stimulatory function of heat-killed Lactobacillus plantarum KCTC 13314BP (LBP). Treatment with LBP significantly increased the production of TNF- $\alpha$ and IL- 6 by macrophages. More importantly, LBP was able to enhance the phagocytic activity of macrophages against bacterial particles. Activation of p38, JNK, ERK, NF- $\mathrm{B}$, and STAT3 was involved in the immunomodulatory function of LBP. LBP treatment significantly increased production of TNF- $\alpha$ by bone marrow-derived macrophages and splenocytes, further confirming the immunostimulatory effect of LBP in primary immune cells. Interestingly, the immunomodulatory effects of LBP were much stronger than those of Lactobacillus rhamnosus GG, a well-known probiotic strain. These results indicate that LBP can be a promising immune-enhancing functional food agent.

Keywords: Lactobacillus plantarum, immunomodulatory effect, phagocytosis, macrophages

\section{Introduction}

Macrophages play a pivotal role in the innate immune system, by protecting the host against pathogens and various diseases [1]. Activated macrophages secrete cytokines, such as tumor necrosis factor- $\alpha$ (TNF- $\alpha$ ), interleukin-6 (IL6) and interleukin-1 (IL-1) to regulate host defense and inflammation. They can also produce various factors including enzymes, complement proteins, proteinases (elastase, collagenase, and urokinase), and nitrogen/ reactive oxygen species to control tissue homeostasis [1-3]. Macrophages are considered professional phagocytes. Phagocytosis by macrophages is crucial for controlling infectious agents in the body and contributes to tissue remodeling, immune responses, and inflammation [4]. Phagocytic activation can regulate cell status, and macrophages can scavenge bacteria, viruses, dead cells, and tumor cells via phagocytosis [1, 2].

Mitogen-activated protein kinases (MAPKs) including extracellular signal-regulated kinases-1/2 (ERK-1/2), p38 MAPKs, and c-Jun N-terminal kinase/stress-activated protein kinases (JNK/SAPKs) have been known as signalling pathways which are related to macrophage activation [5]. Many studies have shown that MAPKs are up-regulated in RAW264.7 macrophages by various inducers such as lipocortin 1 (annexin 1), lipopolysaccharides (LPS), Mycoplasma fermentans, lipoproteins, hyperoxia, and TNF [6]. ERK1/2 and p38 control the expression of genes 
involved in innate immunity. JNK1 can regulate inducible NO synthase (iNOS) [7]. Nuclear factor kappa-light-chainenhancer of activated B cells (NF-kB) plays an important role in macrophage activation via stimulation of cytokine production $[8,9]$. NF- $\mathrm{KB}$ can induce the transcription of genes encoding the cytokines TNF- $\alpha$, IL-6, IL-1, and interferon-gamma (IFN- $\gamma$ ) and control the acute phase of the inflammatory response to maintain homeostasis [10].

In recent years, studies have demonstrated that probiotic microorganisms, which are inactivated by heating, ultraviolet (UV) light, and sonication, have immunomodulatory activities in in vitro and in vivo [11]. Probiotics have also been reported to have bioactivities against atopic diseases $[12,13]$, colic $[14,15]$, diabetes $[16,17]$, and infections [18, 19]. Lactobacillus is a genus in the group of lactic acid bacteria (LAB) [20]. Lactobacillus plantarum is commonly used for fermentation in the dairy industry [21,22] and has been reported to have various immunomodulatory effects, including activation of the Th1 immune response [23], prevention of infections [24], control of allergic diseases [25], and activation of natural killer (NK) cells [26].

There have been no studies on the bioactivity of Lactobacillus plantarum KCTC 13314BP (LBP). Thus, in the current study, we investigated the immunomodulatory effect of LBP and examined the underlying molecular mechanisms.

\section{Materials and Methods}

\section{Materials}

Antibodies for phospho-p38, phospho-ERK, phospho-JNK, phospho-NF-кB, phospho-STAT3, JNK, and STAT3 were purchased from Cell Signaling Technology (USA). Antibodies for ERK, p38, and vinculin were obtained from Santa Cruz Biotechnology (USA). Lactobacillus plantarum KCTC 13314BP was isolated from kimchi, which was purchased from a local market. Lactobacillus plantarum KCTC 13314BP is a strain deposited to the Korean Collection for Type Cultures (KCTC), and its characteristics were examined based on $16 \mathrm{~S}$ rRNA gene sequence and the API 50 $\mathrm{CH}$ test. Lactobacillus rhamnosus GG KCTC5033 was purchased from the KCTC (Korea).

\section{Methods}

Preparation of heat-killed Lactobacillus plantarum KCTC 13314BP (LBP) and Lactobacillus rhamnosus GG KCTC5033 (LGG). LBP and LGG were pre-cultured in $10 \mathrm{~mL}$ of MRS broth supplemented with $2 \%$ maltose (mMRS) at $30^{\circ} \mathrm{C}$ for $24 \mathrm{~h}$. Cultures of LBP and LGG in $5 \mathrm{~L}$ of mMRS were prepared by repeating this cultivation process. After these incubation processes, LBP and LGG were isolated by centrifugation. Distilled water was added to isolated LBP and LGG to achieve a moisture content of $90 \%$.
Then, LBP and LGG were sterilized by heat-treatment at $121^{\circ} \mathrm{C}$ for $15 \mathrm{~min}$.

Cell culture. RAW264.7 murine macrophage cells were purchased from the Korean Cell Line Bank. Cells were cultured at $37^{\circ} \mathrm{C}$ in Dulbecco's Modified Eagle Medium (DMEM, Welgene, Korea) supplemented with $10 \%$ fetal bovine serum (Gibco, USA) and $1 \%$ penicillin and streptomycin (Corning, USA) in a humidified chamber with $5 \% \mathrm{CO}_{2}$.

Enzyme-linked immunosorbent assay (ELISA). TNF- $\alpha$ and IL6 levels in cell culture media were quantified by ELISA following the manufacturer's instruction (R\&D Systems, USA). Ninety-sixwell microplates were incubated with the capture antibody overnight at room temperature (RT). The following day, plates were blocked with $1 \%$ BSA in PBS and washed with wash buffer. Samples and standards were added, followed by a $2 \mathrm{~h}$-incubation. Plates were washed again, and after addition of detection antibody, were incubated for an additional 2 h. After washing, streptavidin-HRP was added for $20 \mathrm{~min}$. After a final wash, the $\mathrm{HRP} /$ substrate reaction was terminated with an equal volume of $2 \mathrm{~N} \mathrm{H}_{2} \mathrm{SO}_{4}$, and absorbance was measured using a Varioskan Lux Multimode microplate reader (Thermo Fisher Scientific, USA) at 450-570 nm.

Cell viability assay. Cell viability measurements were performed according to the manufacturer's instructions (CellTiterGlo Luminescent Cell Viability Assay Kit, Promega, USA). Cells were seeded into white luminescence plates at a density of $4 \times 10^{4}$ cells/well. After $24 \mathrm{~h}$, cells were starved in serum-free DMEM for $24 \mathrm{~h}$, followed by incubation with various concentrations of samples. After a 24 h-incubation, luminescent reagent was added to each well. The plate was shaken on an orbital shaker for $1 \mathrm{~min}$ and incubated at RT for $10 \mathrm{~min}$. Luminescence was measured using a Varioskan Lux Multimode microplate reader (Thermo Fisher Scientific).

Phagocytosis assay. Phagocytic activity measurements were performed according to the manufacturer's instructions (CytoSelect 96-well phagocytosis assay, CellBiolabs, USA). Cells were seeded into 96-well plates at a density of $4 \times 10^{4}$ cells/well, and the plates were incubated for $24 \mathrm{~h}$. Then, samples were added to each well for $1 \mathrm{~h}$, followed by incubation with E. coli particles for $4 \mathrm{~h}$. Absorbance was measured at $540 \mathrm{~nm}$ using a Varioskan Lux Multimode microplate reader (Thermo Fisher Scientific).

NO production. NO concentration was determined by the Griess reaction. Cells were seeded into 96-well plates at a density of $4 \times 10^{4}$ cells/well. After $24 \mathrm{~h}$, various concentrations of samples were added to each well. After $22 \mathrm{~h}, 100 \mu \mathrm{l}$ of cell culture supernatant from each well was added to new 96-well plates and mixed with $100 \mu \mathrm{l}$ Griess reagent (0.1\% N-(1-naphthyl)ethylenediamine dihydrochloride, $1 \%$ sulfanilamide in $5 \%$ phosphoric acid). Plates were shaken on an orbital shaker for $1 \mathrm{~min}$ and incubated at RT for $15 \mathrm{~min}$. Absorbance was measured at $540 \mathrm{~nm}$ using a Varioskan Lux Multimode microplate reader (Thermo Fisher Scientific). Nitrite concentrations were calculated from a $\mathrm{NaNO}_{2}$ standard curve. 
Western blot analysis. Cells $\left(2.25 \times 10^{6}\right.$ cells / well $)$ were rinsed, scraped off the bottoms of wells, and collected in RIPA lysis buffer containing protease and phosphatase inhibitor (Sigma-Aldrich). After centrifugation of the lysate, supernatants were collected and quantified using the Pierce BCA Protein Assay Kit (Thermo Fisher Scientific). Proteins were separated by $10 \%$ SDS-PAGE and transferred to a nitrocellulose membrane (Bio-Rad, USA). After blocking in 5\% skim milk in TBS containing 0.1\% Tween 20 (TBST) for $1 \mathrm{~h}$, the membrane was incubated with the corresponding antibody overnight at $4^{\circ} \mathrm{C}$. After washing with TBST, the membrane was incubated with HRP-conjugated secondary antibody for $1 \mathrm{~h}$, and membranes were visualized using Western Lightning Plus-ECL reagents (PerkinElmer, USA).

Isolation of bone marrow-derived macrophages. Primary macrophages were isolated from the bone marrow of 6-week-old female C57BL/6 mice. Mice were purchased from Young Bio (Seongnam, Korea). Bone marrow cells were isolated from femurs and tibias. Cells were differentiated for 6 days in DMEM/F-12 (Corning, USA) containing fetal bovine serum, $1 \%$ penicillin and streptomycin, and $40 \mathrm{ng} / \mathrm{ml}$ M-CSF (Peprotech, The Netherlands). The medium was replenished on the third day. All experimental protocols were approved by the Institutional Animal Care and Use Committee (SNU-170220-2-2) of Seoul National University, Seoul, Korea

Isolation of splenocytes. Splenocytes were isolated from the spleens of 6-week-old female C57BL/6 mice. Mice were purchased from Young Bio (Korea). C57BL/6 mice were sacrificed, and spleens were removed aseptically and ground in RPMI1640 containing 10\% fetal bovine serum (Gibco) and 1\% penicillin and streptomycin (Corning) by passage through a $40 \mu \mathrm{m}$ cell strainer. After centrifugation, cells were incubated with ACK lysis buffer (Gibco) for $3 \mathrm{~min}$ on ice to lyse the red blood cells. Cells were collected by centrifugation and resuspended in complete RPMI1640 containing 10\% fetal bovine serum (Gibco) and 1\% penicillin and streptomycin (Corning). All experimental protocols were approved by the Institutional Animal Care and Use Committee (SNU-170220-2-2) of Seoul National University, Seoul, Korea.

Splenocyte proliferation assay. Splenocyte proliferation assay was performed according to the manufacturer's instructions (CellTiter-Glo Luminescent Cell Viability Assay Kit, Promega, USA). Splenocytes were seeded into 96-well, white luminescence plates at a density of $2 \times 10^{5}$ cells/well and then cultured in the absence or presence of various concentrations of samples. After $48 \mathrm{~h}$, luminescence reagent was added to each well, and the plates were shaken on an orbital shaker for $1 \mathrm{~min}$, followed by a 10-min incubation at RT. Luminescence was measured using a Varioskan Lux Multimode microplate reader (Thermo Fisher Scientific).

Statistical analysis. Statistical analyses were performed using SPSS software (SPSS Inc., USA). Data are expressed as the mean \pm standard deviation (SD) and analyzed by Student's $t$-test or oneway analysis of variance (ANOVA) followed by Duncan's Multiple
Range Test. A $p$-value of $<0.05$ was used to indicate statistical significance.

\section{Results}

\section{LBP Displays Immunomodulatory Effects in RAW264.7 Macrophages}

TNF- $\alpha$ and IL- 6 were used as biomarkers to investigate the immunomodulatory function of LBP. We used Lactobacillus rhamnosus GG (LGG) for comparison, as it is

A

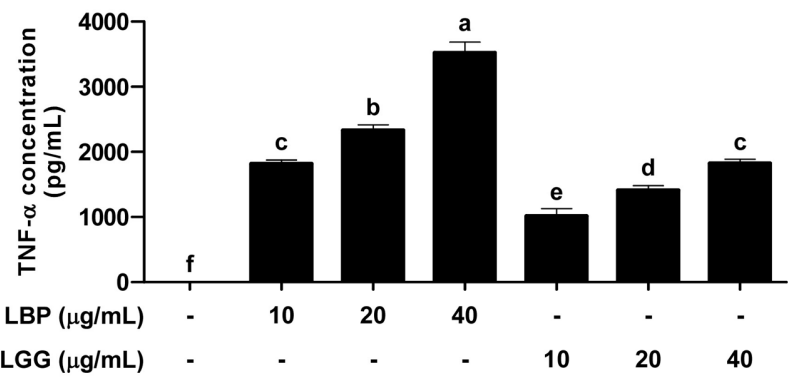

B

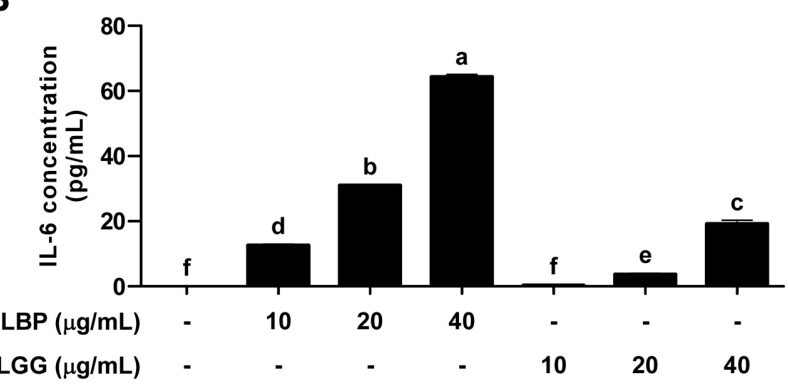

C

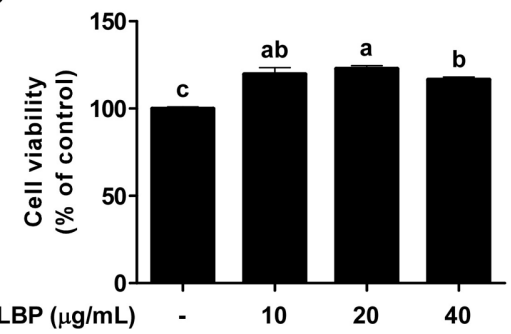

Fig. 1. Effects of LBP on TNF- $\alpha$ and IL-6 production.

(A, B) RAW264.7 macrophages were treated with LBP at the indicated concentrations, and the media were collected after $6 \mathrm{~h}$. TNF- $\alpha$ and IL- 6 levels were measured as described in the Materials and Methods section. (C) RAW264.7 macrophages were treated with LBP for $24 \mathrm{~h}$. Cell viability was measured as described in the Materials and Methods section. Data are represented as mean \pm SD values of three independent experiments. Letters represent significant differences from each other $(p<0.05) .{ }^{* *} p<0.01$, significant difference between E. coli-treated group and E. coli + LBP-treated group. 
widely used as a probiotic agent in the food industry [27]. Treatment with LBP significantly induced TNF- $\alpha$ level in a dose-dependent manner, whereas LGG treatment led to a relatively minimal increase in TNF- $\alpha$ level compared to that of LBP (Fig. 1A). Similarly, LBP also resulted in a greater increase in IL-6 production than did LGG (Fig. 1B). Together, these results suggest that LBP can regulate cytokine production in macrophages.

We also measured the effect of LBP on cell viability. We found that LBP $(10-40 \mu \mathrm{g} / \mathrm{ml})$ treatment caused a moderate increase in cell number compared to the control group (Fig. 1C).

\section{LBP Stimulates Phagocytic Activity and NO Production in RAW264.7 Macrophages}

As phagocytosis is the key feature of activated macrophages against pathogens, we measured the phagocytic activity after LBP treatment. LBP enhanced the phagocytic activity of RAW264.7 macrophages against bacterial particles (Fig. 2A). Recognition of pathogens triggers the
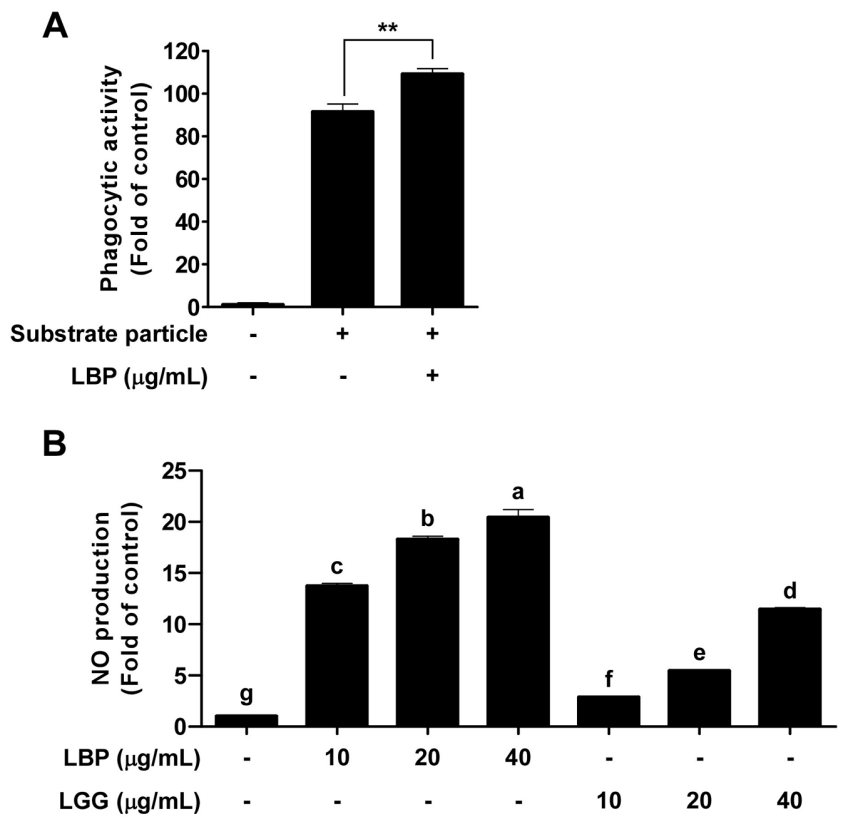

Fig. 2. LBP enhances phagocytic activity and NO production. (A) RAW264.7 macrophages were treated with LBP at $40 \mu \mathrm{g} / \mathrm{ml}$ for $1 \mathrm{~h}$ and phagocytic activity was analyzed as described in the Materials and Methods section. Substrate particles; E. coli. (B) RAW264.7 macrophages were treated with LBP at the indicated concentrations. Media were collected $22 \mathrm{~h}$ after LBP treatment and subjected to NO production analysis as described in the Materials and Methods section. Data are represented as mean \pm SD values of three independent experiments. Letters represent significant differences from each other $(p<0.05)$. activation of macrophages, which in turn can halt pathogen replication by producing NO [28]. We found that LBP increased NO production in a dose-dependent manner (Fig. 2B). LGG treatment also increased NO production compared to the control group but to a lesser extent than LBP (Fig. 2B). LBP, therefore, appears to enhance the function of macrophages demonstrated by the increase in NO production and phagocytic activity.

\section{LBP Regulates p38, ERK, JNK, NF-KB, and STAT3 Signaling Pathways}

To understand the molecular mechanisms responsible for the immunomodulatory effects of LBP, we investigated the major signaling pathways regulating macrophage activation. The MAPK members, p38, ERK, and JNK, as well as transcription factors NF- $\mathrm{kB}$ and STAT3 all play key roles in macrophage activation $[5,6,8,9,29]$. Treatment with LBP activated p38, JNK, ERK, NF-kB, and STAT3 signaling pathways (Fig. 3), suggesting that the immunomodulatory function exhibited by LBP could involve the activation of p38, ERK, JNK, NF-kB, and STAT3 signaling pathways.

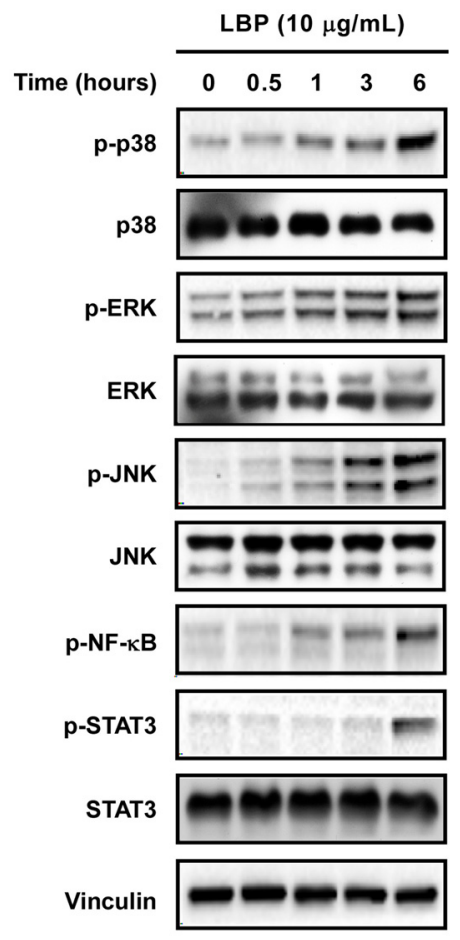

Fig. 3. Involvement of $\mathrm{p} 38, \mathrm{ERK}, \mathrm{JNK}, \mathrm{NF}-\mathrm{\kappa} \mathrm{B}$, and STAT3 in the immunomodulatory effect of LBP.

RAW264.7 macrophages were treated with LBP for 0, 0.5, 1, 3, and $6 \mathrm{~h}$. Cells were lysed, and proteins were subjected to immunoblotting. Vinculin was used as a loading control. 


\section{LBP Shows Immunomodulatory Effect in Primary Macrophages and Splenocytes}

Next, we isolated bone marrow-derived macrophages (BMDMs) and splenocytes from mice to determine if LBP had immunomodulatory effects on primary immune cells. LBP treatment significantly increased TNF- $\alpha$ production by BMDMs and splenocytes in a dose-dependent manner. Notably, LBP treatment resulted in higher TNF- $\alpha$ production than LGG (Figs. 4A and 4B). Additionally, we measured the effect of LBP on splenocyte proliferation. Treatment of splenocytes with LBP led to a significant increase in the number of viable splenocytes (Fig. 4C). Taken together, these results indicate that LBP can generate immune-stimulatory effects also in primary immune cells.

\section{Discussion}

In the current study, we have found that LBP could elicit immune-stimulatory activity in macrophages. LGG is one of the most commonly used probiotic strains and also has been reported to show immunomodulatory effects, including enhancement of immune responses [30, 31]. In our study, LBP stimulated higher cytokine production in RAW264.7 macrophages as well as in primary macrophages compared to that of LGG. Cytokines secreted from innate immune cells play important roles in the regulation of immune responses against pathogens. In particular, macrophages act as the first line of defense against pathogens to maintain tissue homeostasis and activated macrophages locate to the inflammatory area to engage with pathogens [32]. Macrophages produce various inflammatory chemokines and cytokines, such as $\mathrm{NO}$, interleukin family and TNF- $\alpha$ to propagate immune response and protect our bodies from foreign pathogens [33]. Also, our data showed that LBP upregulated NO production and cytokine expression and activated upstream signaling pathways related to macrophage activation. Thus, these results demonstrate that LBP could be helpful in the defense against invading pathogens via macrophage activation.

Macrophages are professional phagocytes which primarily function to eat foreign or potentially harmful matter to protect the host. Proper phagocytic activity by macrophages are critical in controlling inflammation, tissue recovery, and host defense system to prevent immunological disorders [2]. Especially, phagocytosis by macrophages are pivotal in maintaining tissue homeostasis via uptake and subsequent degradation of pathogens and dead cells [4, 34]. We identified that LBP can enhance the phagocytotic activity of macrophages, implying that LBP has potential
A

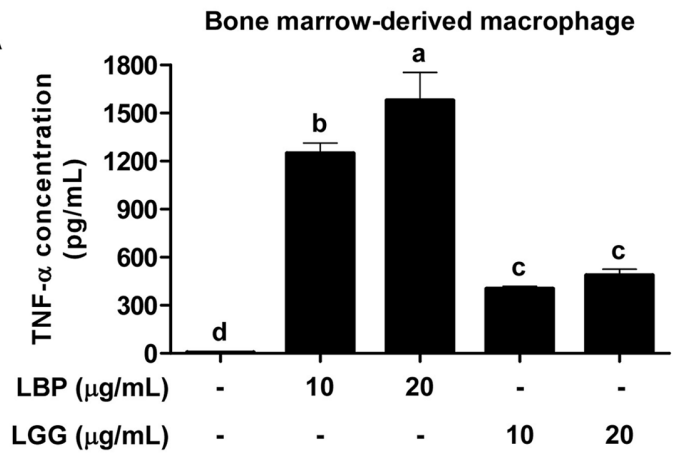

B

Splenocytes

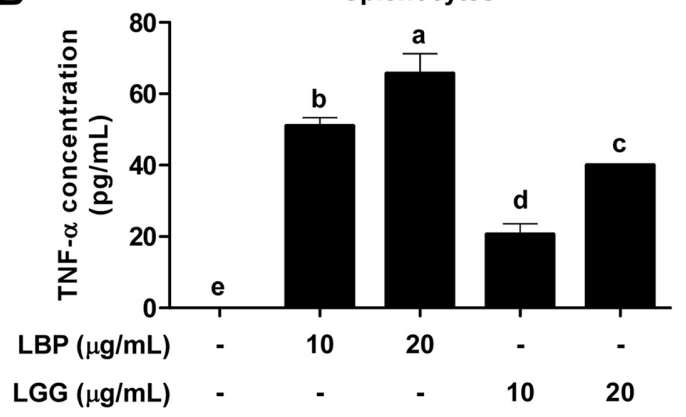

C

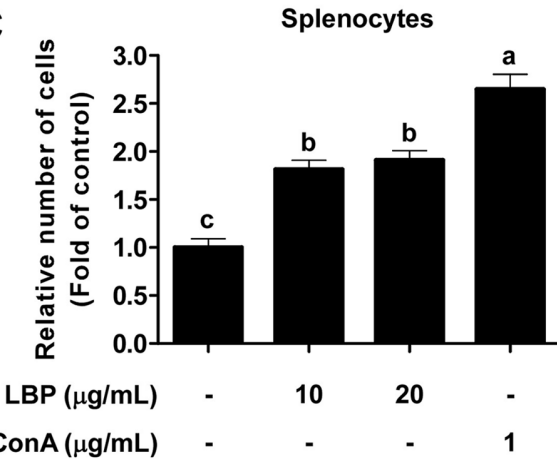

Fig. 4. The effect of LBP on TNF- $\alpha$ production in BMDMs and splenocytes.

(A) Primary BMDMs were isolated from mice and differentiated with m-CSF for 6 days. Media were collected for TNF- $\alpha$ analysis $6 \mathrm{~h}$ after LBP treatment and subjected to ELISA. (B) Splenocyte cells were isolated from mice and seeded in a 96-well plate. Media were collected for TNF- $\alpha$ analysis $18 \mathrm{~h}$ after LBP treatment and subjected to ELISA. (C) Splenocyte cells were isolated from mice and seeded in 96well plates. Celltiter-glo luminescent cell viability assay was performed 3 days after LBP treatment. ConA; Concanavalin A was used as a positive control. Data are represented as mean \pm SD values of three independent experiments. Letters represent significant differences from each other $(p<0.05)$.

for protecting our bodies from foreign pathogens and damaged cells. We also discovered that these immunestimulatory functions of LBP were associated with the 
upregulation of MAPK, NF-кB, and STAT3 signaling pathways.

Although previous studies have reported that certain strains of Lactobacillus plantarum can affect phagocytic activities [35-38], our study demonstrated that LBP can directly enhance the phagocytic activity against E.coli particles in macrophages. In addition, LBP showed superior immunomodulatory activity compared to LGG in RAW264.7 macrophages and primary cells. These immunomodulatory effects of LBP indicate that LBP could help initiate activation of the immune system by increasing cytokine production and phagocytic activity. In conclusion, these results suggest LBP as a potential immune-stimulating agent that can be utilized for the development of novel functional food products.

\section{Acknowledgments}

This project was supported by the SPC group and the National Research Foundation of Korea (NRF) grant funded by the Korea government (MSIP) (NRF-2017R1C1B1006072).

Shin Dal Kang, Seongbong Song, Deukbuhm Lee are employed by the SPC group, and they, along with the other authors, declare no conflict of interest.

\section{Conflict of Interest}

The authors have no financial conflicts of interest to declare.

\section{References}

1. Laskin DL. 2009. Macrophages and inflammatory mediators in chemical toxicity: a battle of forces. Chem. Res. Toxicol. 22: 1376-1385.

2. Gordon S. 2016. Phagocytosis: an immunobiologic process. Immunity 44: 463-475.

3. Wynn TA, Chawla A, Pollard JW. 2013. Macrophage biology in development, homeostasis and disease. Nature 496: 445-455.

4. Aderem A, Underhill DM. 1999. Mechanisms of phagocytosis in macrophages. Annu. Rev. Immunol. 17: 593-623.

5. Lloberas J, Valverde-Estrella L, Tur J, Vico T, Celada A. 2016. Mitogen-activated protein kinases and mitogen kinase phosphatase 1: a critical interplay in macrophage biology. Front Mol. Biosci. 3: 28.

6. Rao KM. 2001. MAP kinase activation in macrophages. J. Leukoc. Biol. 69: 3-10.

7. Valledor AF, Sanchez-Tillo E, Arpa L, Park JM, Caelles C, Lloberas J, et al. 2008. Selective roles of MAPKs during the macrophage response to IFN-gamma. J. Immunol. 180: 45234529.

8. Baeuerle PA, Henkel T. 1994. Function and activation of NFkappa B in the immune system. Annu. Rev. Immunol. 12: 141-179.

9. Grimm S, Baeuerle PA. 1993. The inducible transcription factor NF-kappa B: structure-function relationship of its protein subunits. Biochem. J. 290 (Pt 2): 297-308.

10. Ghosh S, May MJ, Kopp EB. 1998. NF-kappa B and Rel proteins: evolutionarily conserved mediators of immune responses. Annu. Rev. Immunol. 16: 225-260.

11. Taverniti V, Guglielmetti S. 2011. The immunomodulatory properties of probiotic microorganisms beyond their viability (ghost probiotics: proposal of paraprobiotic concept). Genes Nutr. 6: 261-274.

12. Yesilova Y, Calka O, Akdeniz N, Berktas M. 2012. Effect of probiotics on the treatment of children with atopic dermatitis. Ann. Dermatol. 24: 189-193.

13. Doege K, Grajecki D, Zyriax BC, Detinkina E, Zu Eulenburg C, Buhling KJ. 2012. Impact of maternal supplementation with probiotics during pregnancy on atopic eczema in childhood-a meta-analysis. Br. J. Nutr. 107: 1-6.

14. Nation ML, Dunne EM, Joseph SJ, Mensah FK, Sung V, Satzke C, et al. 2017. Impact of Lactobacillus reuteri colonization on gut microbiota, inflammation, and crying time in infant colic. Sci. Rep. 7: 15047.

15. Chau K, Lau E, Greenberg S, Jacobson S, Yazdani-Brojeni P, Verma N, et al. 2015. Probiotics for infantile colic: a randomized, double-blind, placebo-controlled trial investigating Lactobacillus reuteri DSM 17938. J. Pediatr. 166: 74-78.

16. Kadooka Y, Sato M, Imaizumi K, Ogawa A, Ikuyama K, Akai $Y$, et al. 2010. Regulation of abdominal adiposity by probiotics (Lactobacillus gasseri SBT2055) in adults with obese tendencies in a randomized controlled trial. Eur. J. Clin. Nutr. 64: 636-643.

17. Andreasen AS, Larsen N, Pedersen-Skovsgaard T, Berg RM, Moller K, Svendsen KD, et al. 2010. Effects of Lactobacillus acidophilus NCFM on insulin sensitivity and the systemic inflammatory response in human subjects. Br. J. Nutr. 104: 1831-1838.

18. Ahmad K, Fatemeh F, Mehri N, Maryam S. 2013. Probiotics for the treatment of pediatric helicobacter pylori infection: a randomized double blind clinical trial. Iran J. Pediatr. 23: 79-84.

19. Hsieh PS, Tsai YC, Chen YC, Teh SF, Ou CM, King VA. 2012. Eradication of Helicobacter pylori infection by the probiotic strains Lactobacillus johnsonii $\mathrm{MH}-68$ and L. salivarius ssp. salicinius AP-32. Helicobacter 17: 466-477.

20. Seddik HA, Bendali F, Gancel F, Fliss I, Spano G, Drider D. 2017. Lactobacillus plantarum and Its Probiotic and Food Potentialities. Probiotics Antimicrob. Proteins 9: 111-122.

21. Capozzi V, Russo P, Ladero V, Fernandez M, Fiocco D, Alvarez MA, et al. 2012. Biogenic amines degradation by 
lactobacillus plantarum: toward a potential application in wine. Front Microbiol. 3: 122.

22. Arif IA, Bakir MA, Khan HA, Al Farhan AH, Al Homaidan AA, Bahkali AH, et al. 2010. Application of RAPD for molecular characterization of plant species of medicinal value from an arid environment. Genet Mol. Res. 9: 21912198.

23. Kawashima T, Hayashi K, Kosaka A, Kawashima M, Igarashi $\mathrm{T}$, Tsutsui $\mathrm{H}$, et al. 2011. Lactobacillus plantarum strain YU from fermented foods activates Th1 and protective immune responses. Int. Immunopharmacol. 11: 2017-2024.

24. Kikuchi Y, Kunitoh-Asari A, Hayakawa K, Imai S, Kasuya K, Abe $\mathrm{K}$, et al. 2014. Oral administration of Lactobacillus plantarum strain AYA enhances IgA secretion and provides survival protection against influenza virus infection in mice. PLoS One 9: e86416.

25. Rigaux P, Daniel C, Hisbergues M, Muraille E, Hols P, Pot B, et al. 2009. Immunomodulatory properties of Lactobacillus plantarum and its use as a recombinant vaccine against mite allergy. Allergy 64: 406-414.

26. Rizzo A, Losacco A, Carratelli CR, Domenico MD, Bevilacqua N. 2013. Lactobacillus plantarum reduces Streptococcus pyogenes virulence by modulating the IL-17, IL-23 and Tolllike receptor $2 / 4$ expressions in human epithelial cells. Int. Immunopharmacol. 17: 453-461.

27. Segers ME, Lebeer S. 2014. Towards a better understanding of Lactobacillus rhamnosus GG--host interactions. Microb. Cell Fact. 13 Suppl 1: S7.

28. Billack B. 2006. Macrophage activation: role of toll-like receptors, nitric oxide, and nuclear factor kappa B. Am. J. Pharm. Educ. 70: 102.

29. Mosser DM, Edwards JP. 2008. Exploring the full spectrum of macrophage activation. Nat. Rev. Immunol. 8: 958-969.
30. Fong FL, Kirjavainen PV, El-Nezami H. 2016. Immunomodulation of Lactobacillus rhamnosus GG (LGG)-derived soluble factors on antigen-presenting cells of healthy blood donors. Sci. Rep. 6: 22845.

31. Fong FLY, Kirjavainen P, Wong VHY, El-Nezami H. 2015. Immunomodulatory effects of Lactobacillus rhamnosus GG on dendritic cells, macrophages and monocytes from healthy donors. J. Functional Foods 13: 71-79.

32. Parameswaran N, Patial S. 2010. Tumor necrosis factoralpha signaling in macrophages. Crit. Rev. Eukaryot Gene Expr. 20: 87-103.

33. Lee JH, Ahn DU, Paik HD. 2018. In vitro immuneenhancing activity of ovotransferrin from egg white via mapk signaling pathways in RAW 264.7 macrophages. Korean J. Food Sci. Anim. Resour. 38: 1226-1236.

34. Rosales C, Uribe-Querol E. 2017. Phagocytosis: a fundamental process in immunity. Biomed. Res. Int. 2017: 9042851.

35. Butprom S, Phumkhachorn P, Rattanachaikunsopon P. 2013. Effect of Lactobacillus plantarum C014 on innate immune response and disease resistance against Aeromonas hydrophila in hybrid catfish. ScientificWorldJournal. 2013: 392523.

36. Jang SE, Joh EH, Lee HY, Ahn YT, Lee JH, Huh CS, et al. 2013. Lactobacillus plantarum HY7712 ameliorates cyclophosphamide-induced immunosuppression in mice. $J$. Microbiol. Biotechnol. 23: 414-421.

37. Ren D, Li C, Qin Y, Yin R, Du S, Liu H, et al. 2015. Evaluation of immunomodulatory activity of two potential probiotic Lactobacillus strains by in vivo tests. Anaerobe 35: 22-27.

38. Meng Y, Li B, Jin D, Zhan M, Lu J, Huo G. 2018. Immunomodulatory activity of Lactobacillus plantarum KLDS1.0318 in cyclophosphamide-treated mice. Food Nutr. Res. 62: doi: 10.29219 . 